\title{
MedienPädagogik
}

Zeitschrift für Theorie und Praxis der Medienbildung

\section{Twist it once, twist it twice}

\section{Mediale Praktiken zwischen künstlerischer Intervention und informellen Bildungsprozessen}

\author{
Anna Wiehl ${ }^{1}$ (1) \\ ${ }^{1}$ Universität Bayreuth
}

\section{Zusammenfassung}

Dieser Beitrag befasst sich mit dem Potenzial interaktiver dokumentarischkünstlerischer Konfigurationen einerseits sowie den Herausforderungen, die sich im Hinblick auf Bildungsangebote in, mit digitalen und durch digitale Netzmedien auftun andererseits. Dabei werden vor allem drei Aspekte untersucht, die bei der Erschliessung emergierender Praktiken im Kontext medialer Bildung zentral sind: (1) das Changieren von Medien-Erleben in interactive factuals zwischen Subjektivierungs- und Kollektiverfahrungen; (2) das Versprechen zur Partizipation als Teilhabe und Teilnahme; sowie (3) das Potenzial, handelnden Subjekten zu 〈digitaler Souveränität〉 zu verhelfen. Die leitende Fragestellung ist, inwiefern experimentelle, stark prozessual operierende Konfigurationen als informelle bzw. non-formale Bildungsmedien betrachtet werden können und welchen Beitrag sie auf der Meta-Ebene bzw. durch ihr experience design zur Entwicklung von Medienkompetenzen und einem reflektiert-kritischen Blick auf das eigene <digitale Subjekt> leisten. Nach einem kurzen Überblick über selbstreflexive interaktivdokumentarische Konfigurationen und eine medienhistorische bzw. -ökologische Situierung des Phänomens werden zunächst zwei für web-documentaries eher typisch konzipierte Projekte hinsichtlich ihres Beitrags zu digitaler Souveränität untersucht. Diese Projekte basieren primär auf prozeduraler Rhetorik, narrativer 
Immersion und komplex vernetzter Informationsaufbereitung und fördern damit das, was als digital literacy im engeren Sinne bezeichnet werden kann. Abschliessend wird anhand von network effect (USA 2015; Harris und Hochmuth) die These diskutiert, inwiefern dieses ästhetisch radikale, irritierende Projekt als provokativ-paradoxe Intervention das Spektrum klassischer Strategien des Dokumentarischen, aber auch formeller Bildungsprozesse erweitert.

\title{
Twist it once, twist it twice. Media Practices between Artistic Intervention and Informal Media Education
}

\begin{abstract}
This contribution addresses the potential of interactive artistic-documentary configurations as well as the challenges that arise with regard to educational offerings in, with, and through digital network media. The focus lies on three aspects which are essential in the context of media education: (1) the oscillation of media experiences in interactive factuals between subjectivisation and collectivisation; (2) their promise of participation; and (3) their contribution to advancing (media) competences vis-à-vis digital sovereignty, new media literacy and agency. The guiding questions are: To what extent can experimental configurations be regarded as informal/non-formal educational media? And: In how far can they inspire - on a meta level or through their experience design a critically-reflective view of one's own (digital persona)? After a brief overview of self-reflexive interactive-documentary configurations and a media-historical and media-ecological situating of the phenomenon, two projects which are rather typical of web-documentaries will be examined with regard to their contribution to digital sovereignty. These projects are primarily based on procedural-rhetorics, narrative immersion and complexly networked provision of information, thus enhancing what can be described as digital literacy. In a second step, network effect (USA 2015; Harris and Hochmuth) will be used as a paradigm to discuss the potential of aesthetically radical, irritating projects used as provocativeparadoxical interventions to expand the spectrum of classical strategies of the documentary, but also of formal educational processes.
\end{abstract}




\section{Mediale Transformationskulturen und das Dokumentarische im Digitalen}

Unsere Welt und damit unser individuelles wie kollektives (Selbst-)Erleben in dieser Welt sind in einem ständigen Fluss. Digitale Medien - insbesondere digitale Netzmedien, interaktive, algorithmenbasierte Medien und künstliche Intelligenz - haben Eingang gefunden in alle Lebensbereiche, und so verwundert es nicht, dass u. a. Holze und Verständig Digitalisierung als «Metaprozess» (Holze und Verständig 2020, 5) betrachten, vergleichbar der Globalisierung oder dem demografischen Wandel. Wo früher noch Paradigmenwechsel ausgemacht werden konnten, erleben wir aktuell - so Weber - eine «Kollision von Wertmassstäben»; wir befinden uns in einem «komplexe[n] Prozess, bei dem sich sowohl Massstäbe aus der Vergangenheit als auch der Gegenwart oder neuerdings auch aus der erwarteten $\mathrm{Zu}$ kunft wechselseitig durchdringen» (Weber 2011, 35). Weber spricht hier von «medialen Transformationskulturen», womit er einen «spezifischen Prozess einer fortgesetzten, einer fortdauernden durch Medien bewirkten Transformation» bezeichnet - einen "Prozess eines andauernden, unabsehbaren Wandels» (Weber 2011, 35). Dabei komme es zu einer ständigen wechselseitigen Beeinflussung:

«Mediale Transformationskulturen werden vor allem durch medial vermittelte Formen der Kommunikation geprägt und prägen ihrerseits soziale und kulturelle Verhaltensweisen.» (Weber 2011, 35)

Eine besondere Rolle kommt hierbei dokumentarischen Medien zu: Sie versprechen seit jeher, authentische Zugänge zur Welt zu eröffnen - insbesondere in Zeiten des Umbruchs. Seit ihrer Entstehung agieren audiovisuelle Manifestationen dokumentarischer Praktiken auf Basis des immer wieder angeführten Wahrheits- bzw. Wahrhaftigkeitsanspruchs - des documentary truth claims -, sowie der ihnen eigenen visuellen Evidenz - ihrer visible evidence. ${ }^{1}$ Eng mit dem Dokumentarischen verbunden sind Diskurse um Authentizität und Wahrhaftigkeit. Da das epistemische Potenzial des Dokumentarischen sicherlich nicht auf Indexikalität, vermeintlich

1 Dies betont Nichols, dessen theoriebildende Überlegungen zum dokumentarischen Film Titel tragen wie Representing Reality (1991), «The Question of Evidence, the Power of Rhetoric and Documentary Film» (2008) oder Speaking truths with film (2016). 
objektive Faktizität und mimetische Repräsentation reduziert werden darf, versprechen insbesondere dokumentarisch-künstlerische Praktiken, durch ästhetische Erfahrungen Einblicke zu gewähren in das, was sich rein rationalen Diskursen entzieht und was Herzog als «deeper strata of truth», als «poetic truth» bezeichnet (Herzog und Cronin 2002, 301).

Bei alledem sind dokumentarische Praktiken in zweifacher Hinsicht Teil medialer Transformationskulturen: Sie spielen zum einen eine wesentliche Rolle bei der Wahrnehmung und dem Verständnis dessen, was - vereinfacht gesagt - als eine sich transformierenden 〈Wirklichkeit〉 bezeichnet werden kann; und sie unterliegen zugleich selbst diesem generellen medialen Wandel. Dies trifft insbesondere auf Manifestationen des Dokumentarischen im Digitalen zu, da sie - wie noch in der Analyse herausgearbeitet wird - das Potential aufweisen, die besonderen Affordanzen digitaler Netzmedien einzusetzen. So werden Argumentationsstrategien und ästhetisch-rhetorischen Mitteln, die das Filmisch-Dokumentarischen in Form von Repräsentationsmodi bietet, um das Spektrum der Interaktionsmodi erweitert.

\section{Das 〈i in i-docs und das Potenzial emergierender dokumentarisch-künstlerischer Praktiken im Kontext medialer Bildung}

Zunächst gilt es zu umreissen, was im Folgenden unter <emergierenden Praktiken und Phänomenen des Dokumentarischen im Digitalen〉 verstanden werden soll, und was insbesondere Formen und Formate ausmacht, die zum einen im Bereich kultureller Bildung zum Einsatz kommen können, darüber hinaus aber auch Konfigurationen, die als 〈dokumentarischkünstlerische Intervention〉 bezeichnet werden können.

Gaudenzi und Aston, die mit zu den ersten in der scientific community zählen, die sich mit i-docs beschäftigt haben, wählen für i-docs bewusst eine recht weit gefasste Definition:

«Any project that starts with an intention to document the 〈real〉 and that uses digital interactive technology to realize this intention can be considered an interactive documentary» (Aston und Gaudenzi 2012, 125f.). 
Zugleich betonen sie jedoch, dass Interaktivität mehr ist als nur ein «delivery mechanism»:

«[I]nteractivity is seen as a means through which the viewer is positioned within the artefact itself, demanding him, or her, to play an active role in the negotiation of the 〈reality being conveyed through the i-doc.» (Aston und Gaudenzi 2012, 126)

Dabei sind i-docs dem erweiterten Verständnis nach (indeterminiert) (Zimmermann, zit. n. Aston und Odorico 2018, 65) - das heisst, sie sind offen für kreative Herangehensweisen und ein ständiges Befragen der eigenen medialen Identität; sie sind experimentell und weisen häufig Hybridisierungstendenzen auf - u. a. mit Praktiken, die eher aus dem künstlerischen Bereich kommen, aber auch aus der künstlerischen Forschung (artistic research), media activism und media artivism (Kara und Møhring Reestorff 2015) oder dem digital journalism bzw. data journalism. Nicht zuletzt werden auch die Grenzen zu serious games und games for change sowie Doku-Fiktionen immer durchlässiger.

Angesichts dieser Offenheit, Hybridität und Mannigfaltigkeit von idocs verwundert es nicht, dass neben dem am häufigsten verwendeten Terminus interactive documentary (kurz i-doc) eine Vielzahl anderer Bezeichnungen existiert: expanded documentary, transmedia documentary, database documentaries, docugames, web-documentaries, open space documentary, living documentary, immersive documentary usw. Die meisten Bezeichnungen verweisen dabei auf die naheliegende medienhistorische genealogische Herkunft und tragen das Dokumentarische im Namen; zugleich heben sie aber die spezifischen (technologischen) Affordanzen oder den (sozio-politischen) Impetus des 〈Neuen〉 hervor.

Die terminologische Vielfalt spiegelt die Gemengelage wider, aus der heraus i-docs (um hier auf die gängigste Bezeichnung zurückzugreifen) entstanden sind. Sicherlich stehen sie zu grossen Teilen in der Tradition des Dokumentarfilms, dennoch wäre es zu kurz gegriffen, sie einzig als eine Fortsetzung des Dokumentarisch-Cineastischen in einem anderen Medium - in digitalen bzw. Netzmedien - zu sehen. Vielmehr gilt es, die sich gegenwärtig manifestierenden Praktiken aus einer medienökologischen Perspektive heraus zu betrachten, also als Teil eines dynamischen 
Systems, in dem Manifestationen synchron wie diachron auf vielfältige Weise miteinander vernetzt sind. ${ }^{2}$ Digitale Technologien - insbesondere vernetzte, ubiquitäre Medien -, aber auch neue Erzählmodi und Ästhetiken bedingen neue Konzeptionen des Dokumentarischen ebenso wie neue Infrastrukturen, (digitale) Plattformen, eine Tendenz zu datafication und gamification sowie zu veränderten Ansprüchen und Erwartungen der Nutzenden, die sich gerade im Bereich von i-docs immer mehr als Partizipierende, inter-actors und co-creators sehen. ${ }^{3}$ In diesem Sinne oszillieren i-docs im erweiterten Sinne zwischen informellem Bildungs-, Partizipations- und Informationsangebot, künstlerischer Intervention und dem Öffnen eines Raums für die Bildung eines digitalen 〈Ich〉 und eines digitalen 〈Wir〉.

Dieser Beitrag fokussiert drei Problemfelder, die im Kontext medialer Bildung zentral sind, sowie die Frage, inwiefern i-docs einen Beitrag leisten können, das Potenzial und die Herausforderungen, die Handeln in digitalen Netzwerken mit sich bringen, kritisch zu reflektieren.

- Wie steht es in diesem Kontext um das Versprechen eines medial induzierten Erlebens von Subjektivierungs- und Kollektiverfahrungen - bzw. die Interdependenz eines individuellen (Sich-Positionierens) in einer digital vernetzten Welt, eines 〈Sich-in-Beziehung-Setzens) zu anderen (vgl. Beck 1992) und der Konstruktion eines digitalen 〈Wir〉,

2 Dementsprechend ist die Frage, ob i-docs als «evolutionary» oder «revolutionary» (vgl. Nash 2022, 2; Winston, Vanstone, und Wang 2017, 9) im Hinblick auf dokumentarischen Film zu verstehen sind, als wenig zielführend zu bewerten; interessanter hingegen erscheint die Beschäftigung mit den vielfältigen Faktoren, die zu den ebenfalls mannigfaltigen Manifestationen von i-docs und deren unterschiedlichen Implikationen führen und wie deren spezifisches Potenzial in unterschiedlichen Kontexten genutzt werden kann.

3 Im Folgenden werden die Bezeichnungen Nutzende bzw. Interaktantinnen und Interaktanten (oder auch Interagierende) bzw. Partizipierende synonym verwendet. Dabei sei darauf verwiesen, dass auch in der wissenschaftlichen englischsprachigen Fachliteratur mit verschiedenen Amalgamierungen von user, consumer, producer, author, interactor etc. experimentiert wird, die im Deutschen bisher wenig gängig sind. Daher, sowie um wenig eingebürgerte Anglizismen zu vermeiden, soll auf die sonst von der Autorin bevorzugte Formulierung user-interactor verzichtet werden. 
- Wird das Versprechen zur Partizipation und ihr Potenzial, handelnden Subjekten zu einer (im Folgenden noch näher zu bestimmenden) <digitalen Souveränität〉, insbesondere im Kontext von Bildungsprozessen zu verhelfen, eingelöst?

- Und nicht zuletzt: Wird das Versprechen gehalten, dass mithilfe interaktiver digitaler Konfigurationen komplexe Zusammenhänge erfahrbar gemacht werden, was wiederum zur nachhaltigen Auseinandersetzung mit komplexen Themen anregt?

Die Frage, die über den folgenden Überlegungen steht, lässt sich dementsprechend so zusammenfassen:

Ist es möglich, mit interactive documentaries umfassend multimodale Kompetenzen zu vermitteln, die über primäre digital literacies hinaus zu digitaler Souveränität führen, insofern als sie durch ein 〈diving deep〉 ein Problembewusstsein für das häufig im Digitalen zu konstatierende 〈surfing the surface〉 schaffen?

Die leitende These dabei ist, dass $i$-docs prinzipiell in der Lage sind, die Komplexität unserer (digitalen) Welt im Umbruch erfahrbar zu machen, und dass sie mitunter dann das grösste Potenzial entfalten, wenn sie nach dem Motto agieren: 〈twist it once, twist it twice). Dies beinhaltet zum einen, dass sie nicht nur Wissen um die gegenwärtigen Selbst- und Weltverhältnisse oder technische Fähigkeiten vermitteln, sondern dass sie in Form künstlerischer Intervention irritierend und - zugegebenermassen - unbequem und konfrontativ sind. Allerdings beinhaltet es auch, dass sie letztendlich paradoxe Effekte evozieren, um einerseits für die qualitativen Unterschiede zwischen einem Komplexität annehmenden 〈diving deep〉 und einem engagiert-souveränen Handeln in einer Kultur des Digitalen und einer oberflächlich-getriebenen Schein-Partizipation, affektiver und informationeller (Selbst-)Ausbeutung und einem 〈surfing the surface〉 andererseits zu sensibilisieren. 


\section{Subjektivierung, Partizipation und Komplexitätserfahrung in Kulturen der Digitalität}

\subsection{Medien-Erleben zwischen Subjektivierungs- und Kollektiverfahrungen und das Versprechen relationaler Selbstentfaltung}

Wie Holze und Verständig hervorheben, konstituiert sich Subjektivität «in einem unstetigen Feld von subjektivem Innen sowie objektivem Aussen, von individueller Erfahrung, Wahrnehmung und Weltsicht einerseits sowie gesellschaftlichen Strukturen andererseits» (Holze und Verständig 2020, 1).

In medialen Transformationskulturen - insbesondere in einer sich rapide verändernden Welt ubiquitärer, fluider und hochkomplexer digitaler Netzwerke, die als 〈deeply mediatized) (vgl. Hepp 2020) ${ }^{4}$ charakterisiert werden kann - erfährt das Spannungsverhältnis von subjektivem Innen und objektivem Aussen neue Brisanz. Wie kann man sich als Subjekt «sowohl gegenüber sich selbst als auch in der Welt orientieren» (Holze und Verständig 2020,4)? Zentrale Stichworte in allen diese Transformationen begleitenden Diskursen sind 〈Vernetzung〉 und 〈Interaktion〉.

Dabei weisen zahlreiche Praktiken im Digitalen und durch das Digitale Züge auf, die sich auf das Konstituieren einer emanzipatorischen Beziehung von 〈Ich〉 und 〈Wir〉 eher hinderlich auswirken. So beklagt Spengler u. a., dass die «Unterscheidung zwischen off- und online [...] zunehmend brüchig» (Spengler 2020, 69) sei, und dass «Erwartungshaltungen generiert werden, immer erreichbar zu sein und sich informieren zu können sowie klug das Richtige aus einem stetig wachsenden Angebot möglicher Optionen zu wählen». Viele im Digitalen geknüpfte Kontakte, aber auch

4 Mediatisierung beschreibt nach Couldry und Hepp (2013) die Beziehung zwischen dem Wandel von Medien und Kommunikation einerseits, Kultur und Gesellschaft andererseits, wobei dies sowohl qualitative wie quantitative Dimensionen aufweist. 〈Deep mediatization` bzw. 〈tiefgreifende Mediatisierung) stellt dabei ein fortgeschrittenes bzw. umfassendes Stadium der Mediatisierung dar, in dem alle Bereiche des sozialen Lebens untrennbar in digitalen Medien(netzwerken) verwoben sind (vgl. Hepp 2020, 5). Kennzeichnend für sie sind - wie auch in der Analyse der Beispiele immer wieder anklingen wird ein Zuwachs an Konnektivität, die Omnipräsenz von (Netz-)Medien, eine beschleunigende Innovationsdichte sowie eine Datifizierung (fast) aller Lebensbereiche (vgl. Hepp 2018). 
bereits bestehende Beziehungen zeichnen sich häufig weniger durch eine Tiefe und Qualität der Beziehung aus, sondern durch rein quantitatives 〈Mehr〉, 〈Schneller〉, 〈Jederzeit〉 und 〈Überall〉. Dies wiederum führt zu einer oberflächlichen, entfremdenden Virtualisierung von Kollektiv- und Differenzerfahrungen.

\subsection{Das Versprechen von Partizipation und selbstbestimmter Teilnahme}

Ein weiteres Versprechen kultureller Bildung ist individuelle wie gesellschaftliche Emanzipation, welche wiederum die Möglichkeiten zu Partizipation oder besser zu Teilnahme und Teilhabe birgt.

Kulturen des Digitalen werden sowohl im Hinblick auf politische als auch auf soziale und künstlerisch-kreative Teilhabe und Teilnahme häufig mit dem von Jenkins geprägten Schlagwort der participatory culture charakterisiert. Insbesondere auch im Kontext formeller und informeller kultureller Bildung versteht Jenkins participatory culture als eine Kultur, die Vielfalt, Selbstverwirklichung und politische Mündigkeit verheisst (vgl. Jenkins et al. 2009; Jenkins, Ito, und boyd 2016). Damit wäre es zu kurz gegriffen, Partizipation einzig auf instrumentelle Partizipation im Sinne politischer Mitbestimmung einzuengen; vielmehr muss genuin emanzipatorische Partizipation im Zusammenspiel ihrer politischen, gesellschaftlichen und kulturellen Komponenten gesehen werden.

Dabei gilt es allerdings, zweierlei zu bedenken: Zum einen gilt es zu differenzieren zwischen Partizipation in Medien - also die aktive Teilnahme an der Medienproduktion - und Partizipation durch Medien, also «inwieweit mittels Medien eine Beteiligung an öffentlichen Belangen von Kultur und Gesellschaft möglich ist» (Pfadenhauer und Hepp 2014, 240, Herv. AW) - wobei hier die Optionalität und der Einladungscharakter betont werden müssen; und, daran anknüpfend, ist zum Zweiten Partizipation nicht automatisch gleichzusetzen mit einer freigewählten, selbstbestimmten Beteiligung - «a desired connection, an aimed-for transfer of knowledge or an affirmed alliance and presence in other places». Wie Ott herausarbeitet, besteht immer auch die Gefahr, dass die Möglichkeit zur Partizipation kippt und in «harsh separation, involuntary bio- and socio(techno)logical appropriations» (Ott 2018, 5) mündet. 
Fasst man beide Aspekte zusammen, so ist die Basis für souveräne Partizipation - um hier das diesen Artikel perspektivierende Konzept aufzugreifen - geprägt von einem bewusst gewählten Sich-Einbringen sowie einem Mitspracherecht bei zentralen Gestaltungs- und Entscheidungsprozessen.

\subsection{Netzmedien als digitale Wissensräume und das Versprechen von Komplexitätserfassung}

Die damit einhergehende Betonung von Transparenz, Offenheit und informierter Selbstbestimmtheit ist auch ein zentraler Aspekt in der Diskussion des dritten Versprechens, dass häufig im Zusammenhang mit digitalmediatisierter Bildung angebracht wird: das Potential digitaler Medienkonfigurationen, komplexe, dynamische Wissensräume zu öffnen. Dabei werden vor allem die spezifischen Affordanzen digitaler Medien, wie sie u. a. Manovich (2001) beschreibt, ins Feld geführt - meist mit Fokus auf die ihnen inhärente rhizomatische Netzstruktur (Vernetztheit - «networkedness)) und das Potential zur Vernetzung von Akteuren (Vernetzungspotenzial - die Möglichkeit zu 〈networking〉).

Insbesondere digitale, interaktive dokumentarische Projekte berufen sich darauf, dass sie Komplexität nicht nur widerspiegeln und interaktiv erfahrbar gemachen, sondern dass sie zudem wissensgenerierend fungieren. In diese Richtung argumentieren u. a. Aston und Odorico (2018), wenn sie unter Bezugnahme auf das Konzept der Polyphonie feststellen, dass polyphone (bzw. plurivokale, multiperspektivische) i-docs aufgrund ihrer spezifischen soziokulturellen und medialen Affordanzen eine vielschichtige Komplexitätserfahrung jenseits von Binarismen ermöglichen.

In ihrem Plädoyer für mehr polyphon-kreatives Arbeiten «as a means through which we can productively engage with complexity» (Aston und Odorico 2018,66) betonen sie, dass interaktive pluriperspektivische Konfigurationen im Digitalen aufgrund ihrer anti-hierarchischen, offenen, dialogischen, autopoetischen Natur andere Optionen haben als lineare Medien wie z. B. der Dokumentarfilm. Daher sehen sie i-docs als eine Einladung, Perspektivwechsel zu vollziehen: «[they] facilitate a shift between self and other, and between the subjective and the objective» (Aston und Odorico 2018, 72). 
Allerdings ist auch dieses Versprechen differenziert zu betrachten: Komplexitätserfahrungen in non-linearen dokumentarischen Formaten bzw. generell in Angeboten, die zur kulturellen Bildung in ihrem umfassendsten Sinne beitragen möchten, erfordern Aufmerksamkeit, Zeit und die Bereitschaft, sich mit ihnen aktiv auseinanderzusetzen und unter Umständen einen kreativen Beitrag zur Entfaltung von Erfahrungen zu leisten. Sie erfordern einen «non-trivial effort» (Aarseth 1997, 1), wie es Aarseth bezeichnet.

\subsection{Zwischenfazit 1 und Hypothese - Digitale Souveränität und multimodale literacies als Schlüssel zur Realisierung der Versprechen von Subjektivierung, Partizipation und Komplexitätserfahrung im Digitalen}

Fasst man die Überlegungen zusammen, die im Hinblick auf einerseits die Versprechen digitaler Kulturen für digitale kulturelle Bildung diskutiert wurden, und verweist andererseits auf die sie begleitenden Herausforderungen, so ist festzustellen, dass Kulturen des Digitalen nicht nur eine Fülle von Informationen, Vernetzungs- und Partizipationsangeboten bieten; sie sind ebenso gekennzeichnet durch eine Beschleunigung von Wissensproduktion, Fragmentierung, Veroberflächlichung von Beziehungen und zugleich Opazität von algorithmischen Tiefenstrukturen und Hintergrundprozessen, der Ubiquität von verfügbarer Information und einem nie abreissenden Mahlstrom von Kommunikationsflüssen. Die <digital plenitude), wie sie Bolter (2009) positiv konnotiert beschreibt, und das exponenzielle Wachstum von Angeboten führen - kritisch betrachtet - dazu, dass das Bildmotiv des Mosaiks von Informationen und Knotenpunkten nicht mehr in seiner Gänze eingesehen werden kann. So beklagt Spengler, dass mithilfe von Algorithmen zwar «gefilterte Ausschnitte, die übersichtlicher sind und leichter bedient werden können, angezeigt werden - deren basale Funktionsprinzipien sich jedoch dem allgemeinen Verständnis wie der Einsicht entziehen» (Spengler 2020, 68). Dieses Verständnis wäre jedoch zu essenziell, um eine eigenständige Einordnung, Einschätzung, Kontextualisierung und Bewertung vornehmen zu können und um sich dann dementsprechend zu Inhalten positionieren zu können. 
Spätestens an diesem Punkt stellt sich die Frage nach digitaler Souveränität - womit ein Konzept aufgerufen ist, welches mindestens ebenso vielschichtig ist wie das der Digitalität selbst; welches neben einer politischen, juristischen und wirtschaftlichen auch eine kulturelle Dimension aufweist (vgl. u. a. Misterek 2017; Kergel 2018; Arbeitspapier Schlüsselaspekte Digitaler Souveränität der Gesellschaft für Informatik 2020); welches gesamtgesellschaftlich wie individuell betrachtet werden kann; und welches, wie der AKTIONSRAT BILDUNG hervorhebt, sich aus der Verzahnung von technischer Souveränität und ethisch-reflexiver Souveränität ergibt. Neben einem kompetenten Handeln im Hinblick auf Sicherheitsaspekte und einer Einschätzung von Chancen und Risiken, die die Nutzung digitaler Medien mit sich bringen, geht es also darum, «die Wirkungen des eigenen Handelns nicht nur zu kennen, sondern auch reflexiv zu bewerten» (Blossfeld et al. 2018, 24).

Digitale Souveränität kann daher - auch wenn sie definitiv zu Teilen auf der Einstellungs- und Handlungsebene des Einzelnen zu verorten ist - nicht einzig individuell verstanden werden: Vielmehr stellt sich digitale Souveränität als die Summe einerseits der Fähigkeiten und Möglichkeiten einer Person dar, ihre Entscheidungen, die mit digitalen Medien zu tun haben oder von ihnen abhängen, kompetent, selbstbestimmt und sicher umzusetzen; und andererseits benötigt es für die Verwirklichung digitaler Souveränität eine rechtliche, ökonomische, politische - vor allem aber ethische Rahmung, die eine Realisierung dieser Souveränität erlaubt und befördert. Wie Müller, Thumel, Potzel und Kammerl (2020) betonen, ist digitale Souveränität stets relational zu sehen, eingebettet in komplexe soziale Kontexte, womit sie in untrennbarer Interdependenz mit technischen und gesellschaftlichen Bedingungen steht. ${ }^{5}$ Dabei wird zudem immer wieder darauf verwiesen, dass digitale Souveränität über rein

5 Dies spiegelt sich $u$. a. auch in der differenzierten Aufgliederung verschiedener Wissens-, Kompetenz- und Bildungsebenen im Hinblick auf digitale Souveränität wider, wie sie in dem Positionspapier des AKTIONSRATs zu finden ist. Dort erfolgt die Aufteilung in das Feld «technische Souveränität» und «ethisch-reflexive Souveränität», wobei im Falle der letzteren insbesondere in Formulierungen wie «persönliche Souveränität als individuell und gesellschaftlich verantwortungsvoller Umgang [mit digitalen Medien]» die Interdependenz bzw. Relationalität von verschiedenen Dimensionen digitaler Souveränität deutlich wird (vgl. Blossfeld et al. 2018, 18). 
funktional verstandene digital literacy hinausgeht - also weit umfassender ist als 〈Schreib- und Lesekompetenzen im Digitalen〉 oder sogenannte ICTliteracy, die primär technische Anwendungskompetenzen im Hinblick auf Datensicherheit und 〈Sicheres Surfen im Netz〉, Programmierkenntnisse oder ein Verhalten entsprechend der ungeschriebenen aktuell geltenden Netiquette in den Vordergrund stellt.

Vielmehr handelt es sich um die Befähigung, das eigene Potential zu entfalten und somit gesellschaftliche Teilhabe und Teilnahme zu verwirklichen (vgl. Müller et al. 2020, 31). Mihailidis spricht sich daher in diesem Zusammenhang dafür aus, Massnahmen zur digitalen Bildung und zur Erlangung digitaler Souveränität im übergeordneten Kontext von civic literacies zu konzipieren. Eine solche Perspektive bezieht auch gesellschaftlich-perspektivierte Überlegungen mit ein, die neben Lese- und Ausdruckkompetenzen insbesondere ethische, soziale und emotionale Kompetenzen einschliessen: «value systems of agency, caring, persistence, critical consciousness, and emancipation» (Mihailidis 2018, 154).

Nicht zuletzt scheint daher die Forderung logisch und konsequent zu sein, dass Diskurse zu digitaler Souveränität und digitaler Bildung nicht nur gleichermassen technische, rechtliche und soziale Rahmenbedingungen am Rande mitdenken; vielmehr sollten in formellen wie informellen Bildungskontexten verstärkt Angebote zur Erlangung digitaler Souveränität gemacht werden, die darauf abzielen, die Chancen für eine selbstbestimmte Mediennutzung zu erhöhen und die Gleichverteilung von Partizipationsmöglichkeiten zu ermöglichen (vgl. Müller et al. 2020; vgl. auch Blossfeld et al. 2018); vor allem aber sollte es darum gehen, emotionale, soziale und ethische Kompetenzen zu stärken und anzuregen, den Blick auf die soziokulturell komplexe medienökologische conditio humana zu lenken. 


\section{Beiträge zu new media literacy in Form personalisierter Lernerfahrungen oder: (twist it once)}

An dieser Stelle setzen viele interactive documentaries an und wählen dabei - wie es in der Natur des weiten Spektrums von Phänomenen liegt - mannigfaltige Wege.

Zwei Konfigurationen, die unterschiedliche 〈Spielformen〉 der am häufigsten angewendeten Strategien erkennen lassen, sollen im Folgenden kurz vorgestellt werden.

In netwars / out of CTRL (D 2018, A: Thiele, P: Miiqo Studios und filmtank, ZDF/arte) wird in einer fiktionalisierten, aber auf Fakten basierenden Dokumentation über die Gefahren von Cyberangriffen und fehlender digitaler Datensicherheit aufgeklärt.

Die web documentary do not track (CAN, D, F 2015, A: Gaylor, P: arte, BR, National Film Board of Canada, Upian) zielt darauf, mit informativen modularen, personalisierten Einheiten auf die Konsequenzen unreflektierten Agierens mit und in digitalen Netzmedien zu sensibilisieren. ${ }^{6}$

Die dritte hier analysierte Konfiguration schlägt einen anderen Weg ein und soll als eines der konsequentesten, mithin 〈radikalsten〉 Projekte in diesem Kontext untersucht werden. In network effect manifestiert sich gewissermassen die Strategie eines Doppel-Twists von reflexiver Erfahrbarmachung. In der Form der paradoxen Intervention und Irritation versuchen

6 Kriterien für die Wahl der beiden web documentaries waren zum einen das Potential, welches sie bieten, paradigmatische Strategien konzise herauszuarbeiten, aber auch die Reichweite, Rezeption sowie die Verwendung in formellen wie informellen Bildungskontexten - u. a. im Rahmen von Seminaren des Goetheinstituts, als Empfehlung der Bundeszentrale für Politische Bildung und im schulischen Kontext. Zudem wurden die beiden web documentaries mit zahlreichen Preisen ausgezeichnet, wofür Kriterien entscheidend sind, die Aspekte wie ästhetische Innovation und edukativen (Mehrwert) verbinden. [D]o not track wurde u.a. ausgezeichnet mit dem Preis der International Documentary Association, dem Prix Gémaux für die beste Interaktive Reihe, dem International Association of Broadcasters Online Factual Prize, dem Deutschen Preis für Onlinekommunikation sowie dem Preis für crossmediale Programminnovation von Radio Bremen und der Bremischen Landesmedienanstalt; netwars / out of CTRL erhielt u. a. den Grimme Online Award 2015 in der Kategorie «Wissen und Bildungy, den Prix Italia, den South by Southwest Interaction Innovation Award und den Preis für crossmediale Programminnovationen des rbb.Vor allem aber führen bei web documentaries die zentralen in diesem Beitrag eingangs diskutierten Aspekte zusammen mit Blick auf das Verhältnis von Subjekt, Gesellschaft und Netzwerk, Kultur, Bildung und Macht. 
die Medienkünstler Harris und Hochmuth, erfahrbar zu machen, was in wissenschaftlichen Diskursen nur mittels sehr differenzierter Theorien und selbst dann nur schwer erfassbar ist, und was noch viel schwieriger in einem informellen Kontext vermittelt werden kann - was aber zugleich doch ein Kernelement umfassender digitaler Souveränität, emanzipierter Subjektivierung und zugleich partizipativer Kollektiverfahrung darstellt.

[N]etwork effect ist zwar im Grenzbereich von web-Dokumentarismus, Medienkunst bzw. Medienaktivismus zu verorten, aber gerade aufgrund seines ästhetisch-experimentellen paradox-interaktiv-agierenden Charakters und damit der Erweiterung der Ausdrucksformen des Dokumentarischen kann es potenziell - so die Hypothese - auch eine Erweiterung des Repertoires an Strategien digitaler kultureller Bildungsangebote darstellen.

\section{1 (Twist it once〉 zum Ersten: Die Strategie prozeduraler Rhetorik und narrative Immersion in der transmedia documentary netwars / out of CTRL - «There is nothing virtual about this reality"}

In der transmedia documentary netwars / out of CTRL (D 2018, A: Thiele, P: Miiqo Studios und filmtank, ZDF/arte), einer spekulativen, aber auf Fakten basierenden Dokumentation über die Gefahren von Cyberangriffen, ist ein aufklärender Impetus ganz dezidiert zu finden. netwars erzählt nicht nur über verschiedene Medien hinweg «was geschehen könnte - sondern auch, was bereits passiert», wie es in der Beschreibung auf der Website heisst. Die Eröffnungsfrage auf der Startseite des Projektes - «Bist du bereit, deine Sicherheit auf die Probe zu stellen?» - setzt das Motto für die folgenden fünf interaktiven Szenarien, in denen u.a. die Bereiche Cyberkrieg, Datensicherheit und Computerkriminalität thematisiert werden. Auffallend dabei ist, dass die Autorinnen und Autoren auf eine Doppelstrategie in der web documentary setzen - und diese nochmals einbetten auf einer Seite mit einem sehr direkt edukativ-informativen textbasierten Informationsteil, der eindeutig als Bildungsressource gestaltet ist. 
Die zentralen, in der web documentary verwendeten Strategien setzen auf unterschiedlichen Ebenen an. Die eine auf der des Ikonografisch-Assoziativen, indem Bilder, die im kulturellen Gedächtnis verhaftet und stark emotional konnotiert sind, zur Schaffung einer atmosphärischen Dichte beitragen. Hinzu kommt eine stark immersiv-narrative Ausgestaltung der cut scenes. ${ }^{7}$ Die andere Strategie ist auf der Ebene der Spielmechanik und des Prozeduralen zu lokalisieren. Sie kann auch als eine Form von game based learning bzw. gamification betrachtet werden. ${ }^{8}$

Bereits in der Eröffnungssequenz des ersten Kapitels spricht Nicolai Kinski die Nutzenden an. Er fungiert als 〈Guide〉 durch die «digital darkness》, wie er es formuliert. Auf das in die Kamera gerichtete «Hello, friend!» folgt eine kurze Einführung in die Dimensionen digitaler Kriegsführung als Konsequenz der (meist freiwilligen) Aufgabe digitaler Souveränität: Cyberwar - so die hier immer wieder aufgerufene Analogie - stehe den beiden Weltkriegen an Grausamkeit und konkreter Bedrohlichkeit in nichts nach. Zwischen bereits sehr bedrohlich wirkenden close ups bis hin zu Detailaufnahmen Kinskis, die ästhetisch an die Matrix-Filme der WachowskiGeschwister angelehnt sind, wird immer wieder für ein paar Sekunden Archivmaterial eingeschnitten - u. a. Bilder der Grabenkämpfe des Ersten Weltkrieges, Aufnahmen der Massenvernichtung im Zweiten Weltkrieg (beides in Schwarz-Weiss). Das argumentative Crescendo der Sequenz gipfelt in dem etwas länger eingespielten Ausschnitt historischer Filmaufnahmen des mittlerweile ikonischen Abwurfs der ersten Atombombe über Japan, die alleine schon durch ihre Helligkeit und grelle Farbigkeit herausstechen. Durch diese Assoziationsketten sollen die Nutzenden das Ausmass der sonst häufig abstrakt bleibenden Gefährdung erkennen.

7 Der Begriff (cut scene) wird insbesondere in der Computerspiel-Wissenschaft verwendet und bezeichnet filmische Zwischensequenzen in ansonsten interaktiven Konfigurationen, in denen ohne eine direkte physisch-interaktive Beteiligung der Spielenden Inhalte narrativ vermittelt werden.

8 Streng genommen gilt es zu unterscheiden zwischen gamification, game-based learning, games, games for learning und serious games (vgl. Becker 2017). Allen gemein ist jedoch, dass die Spielmechanismen in einem Kontext eingesetzt werden, der über reine Unterhaltung hinausgeht und sie einen edukativen Mehrwert haben. Unterschiede finden sich jedoch im Grad der Ausgestaltung edukativer Ziele, im Kontext des Spielens und in der primären Funktion des Spiels (vgl. u. a. auch Salen 2008; Fuchs et al. 2014; Bogost 2007; 2008). 

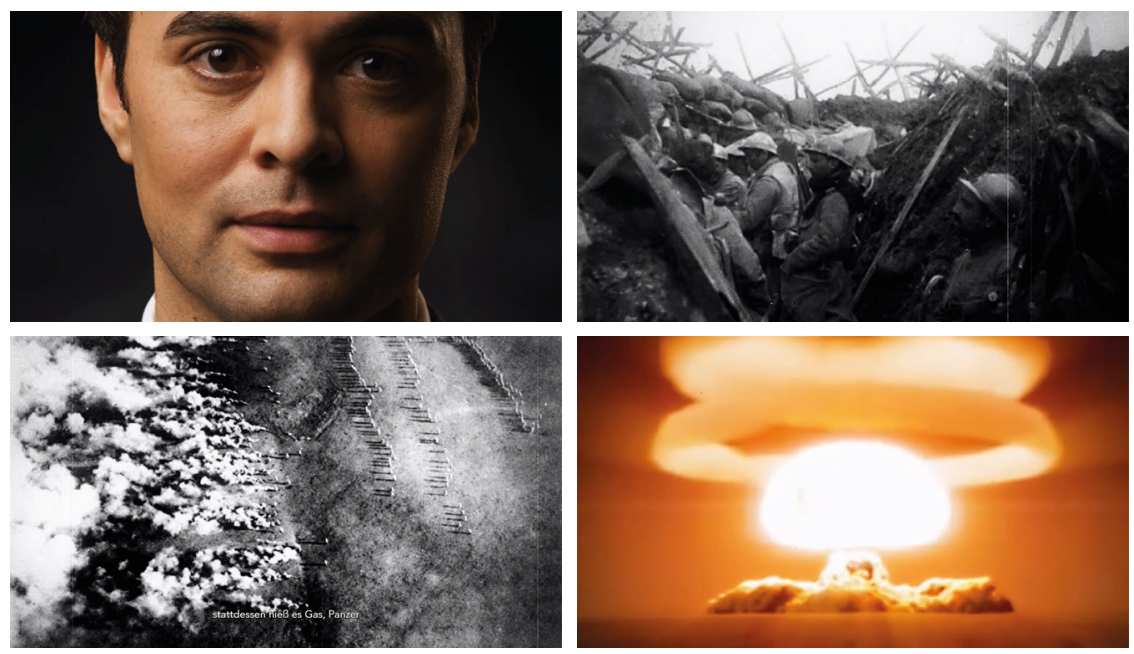

Abb. 1: Screenshots aus netwars - Web Documentary, Episode 1 (Thiele 2015; http:// d3nlbe7zdnif8r.cloudfront.net/all/nw_elal.mp4 in http://www.netwarsproject.com/de/webdoc/episodel; oben links: TC o0:04; oben rechts: TC o०:40; unten links: TC 00:38, unten rechts: TC 00:53).

In der Eingangssequenz stellt sich die Erzählfigur, verkörpert von dem Schauspieler Nicolai Kinski, den Nutzenden als ihr «Guide〉 durch die «digital darkness» vor. Mit einem «Hello, friend!» spricht er die Interagierenden direkt an - wobei sich schon innerhalb der nächsten Minuten herausstellt, dass er ein ziemlich zweifelhafter Freund sein wird.

Im weiteren Verlauf der Eingangssequenz wird das Thema digitaler Souveränität bzw. ihres Fehlens in den Zusammenhang mit cyber warfare gestellt, wobei die titelgebenden netwars noch vernichtender werden dürften als die mit kurzen Einschnitten von Archivmaterial assoziativ aufgerufenen zwei Weltkriege.

Dass digitale Kriegsführung sich nicht nur in spektakulären Hackerangriffen auf Regierungen äussert und nicht nur auf globaler, weltpolitischer Ebene zu finden ist, sondern auch jeden Einzelnen betrifft, wird in den folgenden interaktiven Szenen insbesondere durch personalisierte Erkenntnisprozesse induziert. Denn auch wenn Schlagworte wie 〈netwar〉 oder 〈cyberwar〉 dramatisch aufgeladen sein mögen - letztendlich stehen der Verlust oder die Aufgabe persönlicher Souveränität am Anfang einer 
fatalen Verkettung. Datenmissbrauch, Überwachung und Meinungsmanipulation beginnen - so das Narrativ in netwars - bei jeder und jedem Einzelnen und führen zu einem gesellschaftlichen Dominoeffekt.

Um diesen Verlust digitaler Souveränität den Nutzenden vor Augen zu führen, 〈hackt〉 die Konfiguration persönliche Informationen der Nutzenden - bzw. liest diese aus. So entspinnt sich ein digitales Spionage-Spiel, bei dem die Nutzenden zwangsläufig (den Kürzeren ziehen〉, egal, wie sie sich verhalten; denn blockieren sie das Auslesen ihrer Daten (was aber üblicherweise die wenigsten machen dürften, da damit viele weitere bequeme, z. B. Cookie-basierte Optionen des Surfens unterbunden werden), so endet das 〈Spiel〉 bzw. kann sich erst gar nicht entfalten. Das (vorprogrammierte) Scheitern ist somit in die Mechanik der Konfiguration eingeschrieben.

Eindrucksvoll wird den Interagierenden in den Folgesequenzen vor Augen geführt, wie angreifbar sie sind. Mit den Standardeinstellungen der meisten Rechner und Browser wäre es - rein theoretisch - möglich, nicht nur Nutzungsdaten auszulesen, sondern auch Inhalte und Einstellungen zu manipulieren.

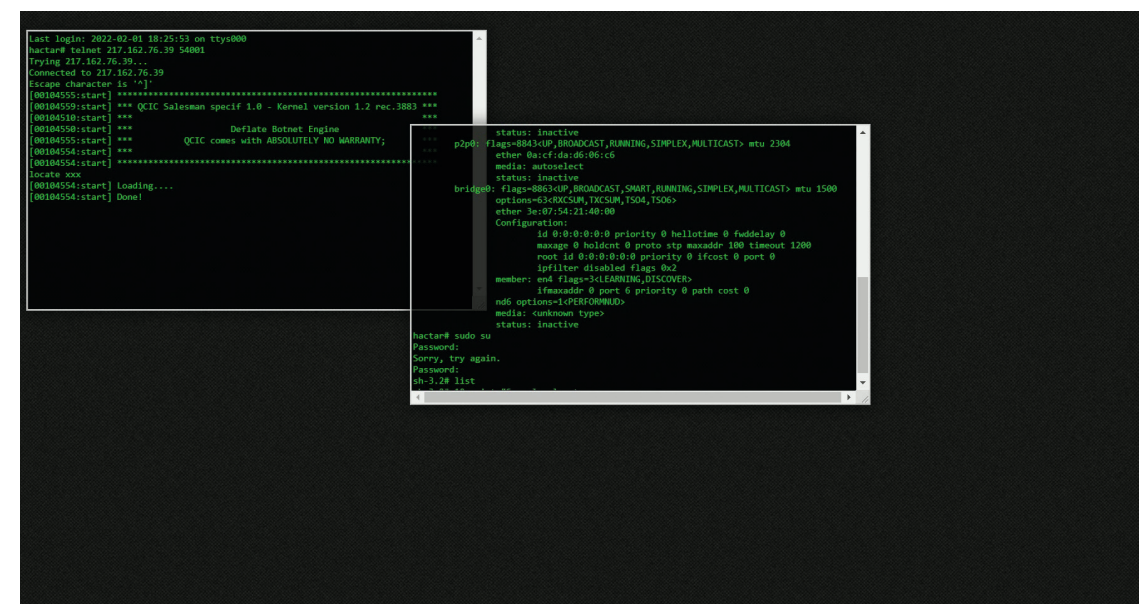

Abb. 2: Screenshot aus netwars - Web Documentary, Episode 1. http://www.netwars-project.com/de/webdoc/episodel/. 
NEWS \& ARTTKEL MAGAZIN GAIN BACKCTRL GLOSSAR

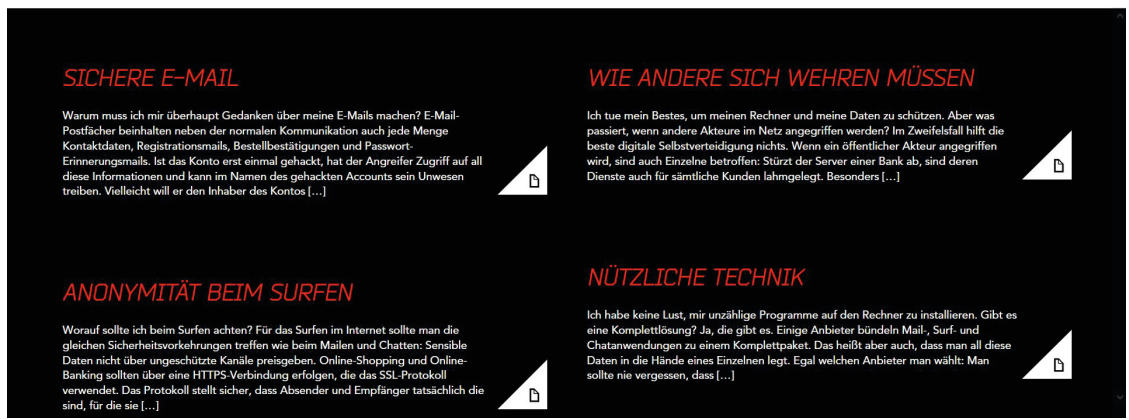

Abb. 3: Screenshot von Screenshot von netwars Projekt-Website 〈Fakten〉. http:// www.netwars-project.com/de/gainbackctrl.

Bereits im ersten Kapitel der web documentary netwars wird den Nutzenden vor Augen geführt, wie gross die Sicherheitslücken der üblichen Standardeinstellungen ihrer Rechner sind und wie weit sie damit die Tore für Späh- und Manipulationsprogramme öffnen. Man beachte hier - neben der Ästhetik, die den Meldungen typischer Trojaner nachempfunden ist den 〈liebevoll〉 gestalteten Tab-Titel (oben).

Direkt im Anschluss an diese Demonstration wird ein weiterer Eintrag im 〈Dossier〉 der Nutzerin oder des Nutzers angelegt: Beim Anklicken des Disketten-Symbols in der Navigationsleiste öffnet sich eine Liste mit personalisierten Zusatzinformationen - je nachdem, welchen Weg man gewählt hat. Diese liefern neben Hintergründen der Recherche praktische Tipps (meist verlinkt), wie die im prozeduralen Spiel von netwars ausgenutzten Sicherheitslücken geschlossen werden können (unten).

Diese Strategie kann als eine 〈Spielform〉 prozeduraler Rhetorik gesehen werden - ein insbesondere in edukativen digitalen Spielen bzw. serious games und news games häufig verwendeter Mechanismus. Das u. a. von Bogost (2007) sowie Treanor und Mateas (2009) beschriebene Konzept der procedural rhetoric (auch prozedurale Rhetorik oder Simulationsrhetorik) beruht darauf, dass Menschen komplexe Zusammenhänge durch eigenes interaktives, regel- und prozessgeleitendes Erkunden unter Umständen schneller und nachhaltiger begreifen können als durch reine Erklärungen oder Demonstrationen. Prozedurale Rhetorik, so Bogost, sei gerade in digitalen Lehr-Lernumgebungen ideal zu realisieren, da sie sich die besonderen 
Affordanzen digitaler Systeme als regelbasierter, responsiver Systeme zu Nutze macht: "Computers run processes, they execute calculations and rule-based symbolic manipulations» (Bogost 2007, ix). Ein besonderes Charakteristikum dieser Form der Rhetorik ist, dass das Prozedurale prozeduraler Rhetorik zum einen das Hervorbringen von 〈Argumenten〉 - also die Vermittlung von Inhalten - betrifft; gleichsam spielt Prozeduralität auch als eine Unterform multimodaler literacies im interaktiven und somit bei Weitem nicht nur rezeptiven Lernen eine Rolle.

«Procedurality refers to a way of creating, explaining, or understanding processes [...] Procedural rhetoric is a technique for making arguments with computational systems and for unpacking computational arguments others have created.» (Bogost 2007, 3)

Um die meist unter der Oberfläche ablaufenden Prozesse sichtbar und erfahrbar zu machen, arbeiten die Autorinnen und Autoren aber nicht nur in den dramaturgisch spannungsreich aufgebauten cut-scenes mit narrativer Immersion und procedural rhetoric. Zwischen die Passagen, in denen sich Kinski direkt als Erzählperson an die Nutzenden wendet, und die Sequenzen, die von den Nutzenden Entscheidungen erfordern (z. B. direkte Fragen) werden immer wieder Expertenstimmen eingebunden. Für die web documentary führten die Autorinnen und Autoren Interviews mit einer Reihe von 〈Cyberexperten〉, deren Perspektive die Nutzenden durch ein Anklicken der Person vertiefen können. Neben einem 〈Steckbrief〉 und dem Video-Statement der jeweiligen Experten finden sich unter dem Reiter «Dossier» weitere Informationen, die insbesondere Verlinkungen zu Quellen enthalten und in denen dann in einer Art 〈Fakten-Check〉 die Aussagen der Interviewpartner belegt, kontextualisiert, aktualisiert, aber auch relativiert werden. 


\section{2 〈Twist it once〉 zum Zweiten: Die Strategie der Transparenz und der Offenlegung von Prozessen in der web-documentary do not track - «Wir wollen Ihnen die Kontrolle zurückgeben»}

Auch die interaktive web-documentary do not track (CAN, D, F 2015, A: Gaylor, P: arte, BR, National Film Board of Canada, Upian) möchte die Nutzenden dafür sensibilisieren, dass die 〈schöne neue Netzkultur〉 zwar komfortabel ist, dass allerdings jede Suchanfrage Datenspuren hinterlässt, mit denen Geschäfte gemacht werden. Dabei setzt auch do not track auf Personalisierung - allerdings anders als dies in netwars der Fall ist: Auf der Basis der Nutzungsdaten der Interagierenden (z. B. IP-Adresse und User-Agent im Moment des Besuchs), die mithilfe der auf den meisten websites gängigen Tools ausgelesen werden, entsteht eine Konfiguration, die eine Form Selbstkonfrontation darstellt. Prozessual erfolgt eine direkte «Aufklärung, die bereits in der web documentary zu einem reflektierten Umgang mit Informationen animieren und somit einen Beitrag zur new media literacy in einem klassisch medienpädagogischen Sinne liefern möchte. Weit weniger spektakulär inszeniert, werden hier meist alltägliche Situationen herausgegriffen - so z. B. der morgendliche, das Frühstück begleitendende Medienkonsum. Auch ist die Figur, die durch die web documentary führt, der Blogger und TV-Moderator Richard Gutjahr, jemand, mit dem sich die Nutzenden identifizieren können. Für die meisten dürfte das positiv konnotierte bekannte Gesicht der Medienszene als role model wirken - und die Tatsache, dass auch Gutjahr 〈dazulernt〉 während der Reise, dürfte dazu animieren, ebenfalls Handlungsweisen zu überdenken und Verhalten zu ändern. Dabei demonstriert auch do not track den Interagierenden, welche oft ökonomischen und auch politischen Interessen hinter data mining, tracking und insbesondere self-tracking zur Selbstoptimierung stehen, und wie so komplexe technologische Konfigurationen der Kontrolle entstehen.

Bereits im ersten Kapitel eröffnet Gutjahr damit, welche Unmenge an Daten er bereits mit dem ersten Anschalten von Netzmedien und Besuch gängiger und renommierter Nachrichtenportale von sich preisgibt, um dann die Nutzenden damit zu konfrontieren, dass auch 〈er〉 (bzw. die Applikation do not track) (weiss〉, in welcher Stadt sich die Nutzenden aktuell aufhalten, wie das Wetter vor Ort ist, welche Tageszeit es ist, mit welchem 
Browser und Computermodell die Seite geöffnet wurde, etc. - und das sind nur die 〈harmloseren〉 Informationen. Narrativ in kurzen, immer wieder durch interaktive Elemente angereicherten Videosequenzen wird den Interagierenden widergespiegelt, wie viele Informationen sie unbewusst teilen - wobei neben Filmclips auch interaktive Infografiken und Animationen zum Einsatz kommen.

Die Journalistinnen und Journalisten hinter dem Projekt formulieren ihr medienpädagogisches Anliegen, einen Beitrag zu digitaler Souveränität zu leisten, in den 〈abouts〉 sehr direkt. Sie möchten 〈uns〉 - den Nutzenden - die Kontrolle zurückgeben:

«Sie sollen verstehen können, welchen Handel Sie eingehen, wenn Sie Ihre Daten gegen kostenlose Information im Internet tauschen. Wir möchten, dass Sie wissen, wann dies ohne Ihre Erlaubnis geschieht, und wir möchten Ihnen zeigen, was Sie dagegen tun können.» (do not track, «Über uns», https://donottrack-doc.com/de/about)

Wie auch netwars setzt do not track dabei auf prozedurale Rhetorik: «Wir möchten, dass Sie nicht nur verstehen, sondern auch erfahren, was Tracking bedeutet» (Website do not track, «Über uns»).

In sieben Episoden wird daher nicht nur Faktenwissen über OnlineProfiling (Episode 3), big data und «Die Welt der Algorithmen» (Episode 5) auf ansprechende, aber informativ fundierte Weise vermittelt; die Nutzenden werden zudem mit ihrem eigenen Suchverhalten in Informationsnetzwerken und sozialen Netzwerken konfrontiert und mit der Tatsache, wie sehr sie selbst unter Umständen in ihrem social media Nachrichtenkonsum in einer Filterblase gefangen sind (Episode 6, «Die Ich-Zeitung»). Zudem bekommen die Nutzenden gespiegelt, welche Flut von Informationen sie mittels der Geolocation-Funktionen und des Datenverkehrs ihres Smartphones über sich preisgegeben haben (Episode 4 - «Der Spion in Ihrer Tasche»). 


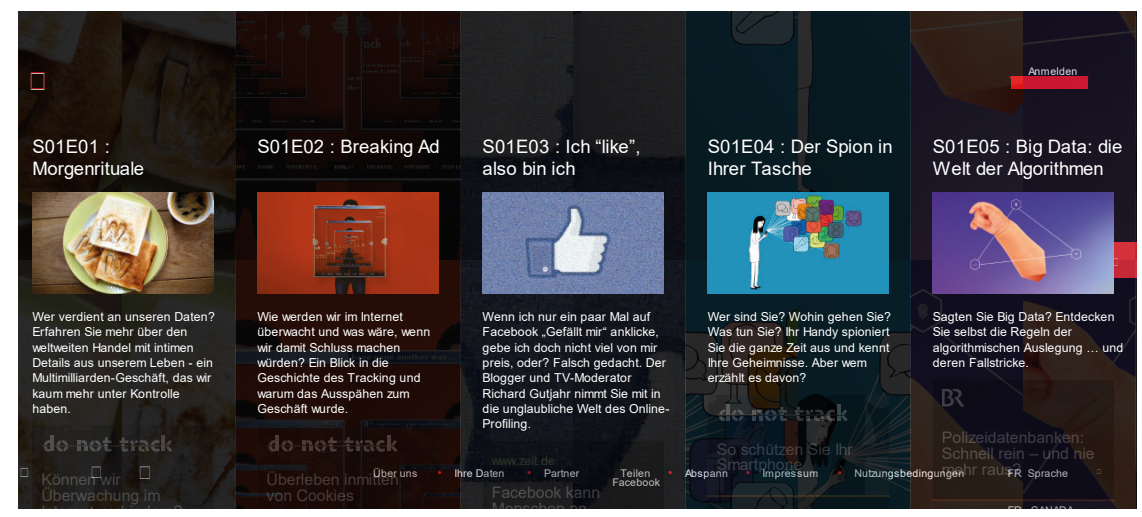

Abb. 4: Screenshot aus do not track (CAN, D, F 2015; https://donottrack-doc.com/ de/episodes/).

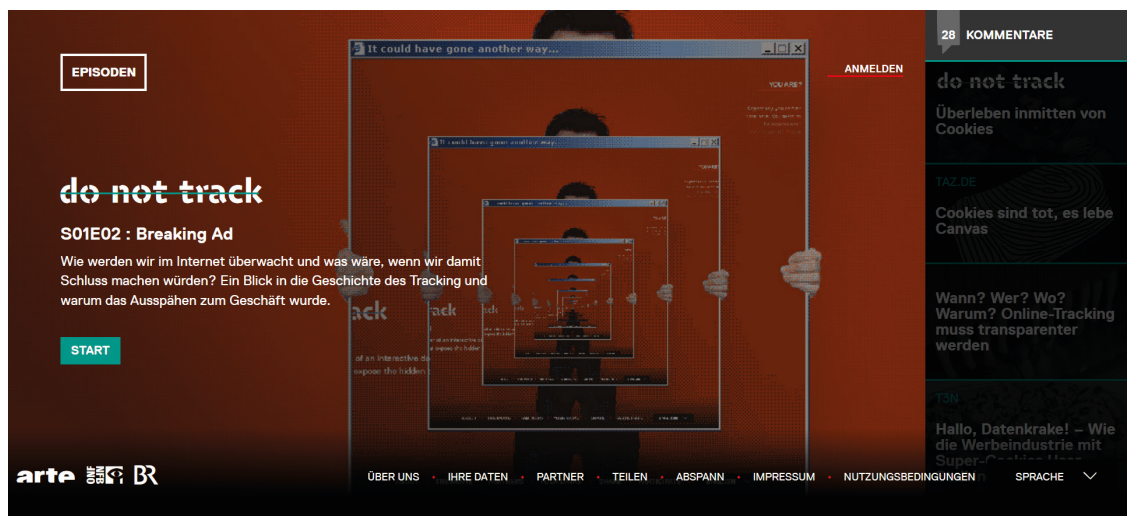

Abb. 5: Screenshot aus do not track (CAN, D, F 2015; https://donottrack-doc.com/ de/episode/2).

[D]o not track klärt in kurzen videobasierten individualisierten Episoden über verschiedene Formen von 〈Macht〉 in digitalen Netzwerken auf (oben). Dabei re-instrumentalisiert die Webseite sowohl technologische Affordanzen als auch visuelle Gestaltungsmittel (hier z. B. die windowingÄsthetik, unten), um die Nutzenden dazu einzuladen, typische Phänomene hinsichtlich ihrer usability bzw. der mit ihnen einhergehenden freiwilligen Unterwerfung unter die Nutzungsmodalitäten zu hinterfragen. 
Generell ist bei dem Projekt - im Gegensatz zu netwars - Transparenz die oberste Maxime. Wo bei netwars die Interagierenden erst im Nachhinein erfahren, warum sie gerade gehackt werden konnten, bekommen sie in do not track bereits im Rahmen der Erzählung die Informationen, die ihnen bewusst machen dürften, dass auch sie angreifbar bzw. manipulierbar sind. Häufig ist es sogar die Erzählperson des Journalisten und Bloggers Gutjahr selbst, die demonstriert, welche Unmenge von Datenspuren er, Gutjahr, hinterlässt, bevor die Nutzenden selbst in einer interaktiven Sequenz zu einem narrativen Knotenpunkt geführt werden, an dem sich die multi-lineare Erzählung dann verzweigt - je nach Wahl der angebotenen Optionen.

Zudem führt der Button in der Fussleiste («Wer wir sind») zu einer Seite, auf der FAQs beantwortet werden und in denen die Nutzenden erfahren, welche Daten wie und wofür genutzt werden, welche Tracking-Tools dabei zum Einsatz kommen etc. Auch wird darüber aufgeklärt, wie Rechercheprozesse abliefen und welche Expertinnen und Experten aus der Informatik-Branche Partnerinnen und Partner des Projekts waren.

\subsection{Zwischenfazit 2: Prozedurale Rhetorik und direkte Erfahrbarmachung als ein erster Twist}

Es kann also festgehalten werden, dass do not track und netwars ähnliche bildungs-kulturelle Ziele verfolgen, dass beide dazu prozedurale Rhetorik verwenden, aber auf unterschiedliche atmosphärische Vermittlungswege setzen.

Zwar wenden sie dazu die gängigen Methoden von Tech-Unternehmen an - jedoch machen sie dies transparent - ob im Vorfeld (do not track) oder im Nachhinein (netwars). Die Interagierenden wissen, worauf sie sich einlassen. Das Verhältnis von Lernprozess, Wissens- und Erkenntniserwerb sowie die Rolle, die dabei die digitalen Konfigurationen in ihrem (Interaktions-)Design spielen, sind von Anfang an klar definiert: Die Nutzenden suchen diese Angebote gezielt auf, weil sie sich Wissensgewinn erhoffen.

Diese Transparenz ist jedoch nicht bei allen Konfigurationen gegeben. Vielleicht aber - so zumindest der abschliessende Impuls dieses Beitrags sind es gerade Projekte, die auf einer anderen Ebene agieren, die mitunter 
das grösste Potenzial bergen, nachhaltig eindrückliche Erfahrungen anzustossen: Konfigurationen, die nicht primär auf der Ebene eines kognitiven Verstehens die Interagierenden ansprechen, sondern vielmehr auf einer affektiven, erlebnisorientierten Ebene. Daher soll im Folgenden, da der Titel dieses Beitrags noch einen zweiten Twist verspricht, der Fokus kurz auf eine dieser anderen Formen von interactive documentaries gelenkt werden - eine Konfiguration, die im Grenzbereich künstlerischer Intervention und digitaler (medien-)kultureller Bildung situiert werden kann; eine Konfiguration, die nicht den Weg der expliziten oder auch edukativ-spielerischen Aufklärung, der Vermittlung von Wissen oder die Anleitung für konkrete Handlungsweisen wählt, sondern die stattdessen versucht, eine paradoxe Erfahrung zu ermöglichen, um so das am Anfang des Beitrags skizzierte Spannungsfeld von 〈Digitalität - Ästhetik - Macht〉 erfahrbar zu machen.

\section{Selbstreflexive dokumentarisch-künstlerische Konfiguration als paradoxe Intervention - oder: network effect als ein (twist it twice)}

[N]etwork effect (USA 2015; Harris und Hochmuth), ein Projekt, das auf irritierende Weise seine eigene epistemologische Wirkmacht und ontologische mediale Identität und Ästhetik inszeniert, instrumentalisiert und zugleich hinterfragt, startet mit einer Art Disclaimer:

«The Internet is said to show our common humanity. Through its data, it is said to provide a kind of omniscience, and through its social networks, a deeper sense of connection. For those without access, it holds the promise of a better life. For those of us who use it a lot, its power to affect our lives is clear - but what is the nature of that effect? How does it change our behavior? The way we see others? The way we see ourselves?» (Harris und Hochmuth, Texttafel beim Aufruf der Website http://networkeffect.io)

Wie Hochmuth und Harris erklären, vermittelt das Internet das Gefühl, allwissend und allumfassend zu sein - was einerseits das Versprechen umfassender Komplexitätserfahrung, ubiquitärer Wissenswelten, grenzenloser zwischenmenschlicher Verbundenheit und Austauschs birgt 
(für die, die über einen technischen Zugang - also 〈access〉 - sowie literacy und die nötigen skills); auf der anderen Seite jedoch birgt eine tiefgreifend mediatisierte und vernetzte Welt das Risiko einer kaum abzuschätzenden Beeinflussung unseres Verhaltens und potenziell eine Manipulation unserer Sichtweisen (auf die Welt), unser Selbstbild und die Art, wie wir Alterität sehen und beurteilen.

Auch wenn das Verhältnis von Subjekt und digitaler Gesellschaft in den bereits besprochenen web documentaries thematisiert wurde (in do not track ganz explizit im Zusammenhang mit Filterblasen in Episode 6, «Die Ich-Zeitung»), so findet die Auseinandersetzung mit der Prekarität von Selbst- und Weltbild in network effect auf einer noch tiefergehenden Ebene statt: Es geht um grundlegende Manipulation unserer Wahrnehmungsprozesse, Aufmerksamkeitslenkung, Denkweisen und vor allem Subjektkonzeption sowie der Konzeption von Gemeinschaft.

Dazu greifen Harris und Hochmuth ein Phänomen unserer vernetzten Gesellschaft auf: das der 〈digital plenitude〉 - also der Flut von Daten und Informationen, Angeboten und Optionen, Kontakten und Kommunikationsströmen, die jedoch häufig nur instantane monologisch-narzisstische Selbstvergewisserungen einer selfie-Kultur darstellen. Dabei spielen Harris und Hochmuth zusätzlich mit dem Druck, den diese Hyper-Komplexität auslöst - der Druck ständiger Verfügbarkeit, (vermeintlicher) Interaktion und Partizipation, wie er im Zwischenfazit 1 beschrieben wurde.

Für network effect haben Hochmuth und Harris über 10.000 Videoclips, 10.000 gesprochene Sätze, Nachrichten, Tweets, Diagramme, YoutubeVideos, Listen und Millionen von individuellen Datenpunkten gesammelt, die sich mit 100 (zwischen-)menschlichen Verhaltensweisen befassen (z. B. 〈hug〉, (argue〉, 〈burn〉, 〈grieve〉, 〈destroy〉 oder 〈love〉) - Verhaltensweisen, die das Verhältnis zum eigenen Sein und dem des bzw. der 〈Anderen〉 beschreiben. 

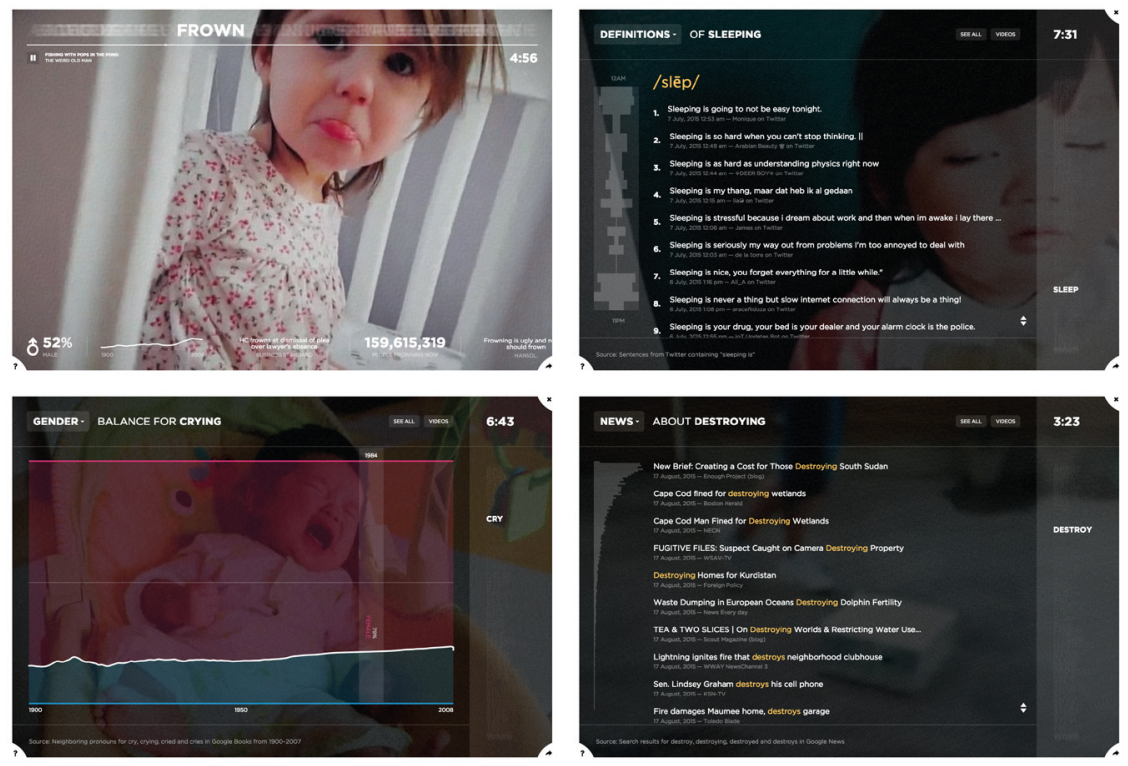

Abb. 6: Screenshots aus network effect (USA 2015; Pressebilder; https://files.network-effect.io/static/assets/v1/assets/press/images.zip?1.40).

Anhand von Stichwörtern mit bestimmten menschlichen Verhaltensweisen und Einstellungen werden digitale Artefakte gesammelt und in interaktiven Info-Grafiken aufbereitet - ohne dass allerdings aus den so visualisierten und untereinander verlinkten Inhalten wirklicher epistemologischer Mehrwert entsteht.

Zur Datenvisualisierung werden all diese Informationen in verschiedenen interaktiven Grafiken zusammengeführt, verlinkt; sie sind untereinander verschlagwortet und mit einer Fülle von Metadaten versehen. Alle Items zu lesen, anzuschauen und anzuhören, würde Tage erfordern - und um die Inhalte der 〈snipets〉 zu kontextualisieren, kritisch distanziert zu lesen und zu durchdenken, wären wahrscheinlich Wochen erforderlich. Hinzu kommt, dass viele Informationen, die sich aus dem Korrelieren von z. B. Youtube Videos mit dem Begriff 〈hug〉 und dem Begriff 〈drink〉 ergeben, wenig Erkenntnisgewinn liefern, und es mag zwar vielleicht die menschliche Neugierde für Kurioses befriedigen herauszufinden, in welchem Jahr mehr Frauen öfters etwas zu 〈strip〉 gepostet haben, wie alt sie waren und woher sie kamen, jedoch ist auch der Mehrwert dieses Wissens mehr als fraglich. Dessen dürften sich aber die meisten Besuchende der Webseite kaum 
bewusst machen, denn dafür ist keine Zeit, ebenso wenig wie für kritische Kontextualisierung oder aber einen verarbeitenden Erkenntnisgewinn. Denn - und das führt zum zweiten Twist -: Beim Betreten der Seite ist die Betrachtungssitzung der Nutzenden auf etwa sieben bis acht Minuten begrenzt (dies entspricht der Umrechnung von Jahren in Minuten bezüglich der zu erwartenden durchschnittlichen Lebensdauer der Nutzenden basierend auf Angaben zu Standort und Geburtsdatum). ${ }^{9}$ Allein schon der Zeitdruck aufgrund der knapp bemessenen möglichen Aufenthaltsdauer auf der Seite erzeugt ein Gefühl des Gehetzt-Seins und schreiender Dringlichkeit. Stets sichtbar läuft ein Countdown mit, das Gefühl der Hektik wird verstärkt durch ein atemlos-aufgeregtes Vorlesen der Tweets, und ein pulsierendes Geräusch im Hintergrund erinnert an einen immer schneller werdenden Puls. Die Angst, etwas Wichtiges zu verpassen, steigt, und mit ihr die Eile, mit der man durch die (meist kurzen) Items hastet. Dies resultiert in einem Gefühl des Überwältigt- und Überfordert-Seins angesichts der Vielzahl und Unüberschaubarkeit der Daten und Informationen. So hinterlässt die Konfiguration nach der zugelassenen Verweildauer auf der Seite den frustrierend-schalen Geschmack, dass zwar die Zeit abgelaufen ist, dass man aber als Nutzerin und Nutzer zu keinem befriedigenden oder erfüllenden Ende gefunden hat. Weder hat man mehr über sich selbst erfahren noch über andere - weder konnten im Sinne von Subjektivierungsprozessen Erkenntnisse über einen selbst gewonnen werden, noch konnte eine Beziehung zu 〈anderen〉 aufgebaut werden - von einer Kohärenz- oder Selbstwirksamkeitserfahrung ganz zu schweigen. Post-strukturalistisch gesprochen lässt sich feststellen, dass es in Konfigurationen wie network effect bzw. den ihnen zugrunde liegenden alltagsweltlichen medialen Praktiken nur permanente différence/différance gibt, und dies in Derridas doppeltem Sinn von «Unterschied» und «Bedeutungsaufschub». Ein Gefühl für das 〈grosse Ganze〉 in seiner durchaus auch konstruktiv anzunehmenden Komplexität bleibt aus.

9 Die approximative Rechnung basiert auf der Selbsterfahrung der Autorin, Jahrgang 1981, und ihren Angaben beim Aufrufen der Seite. 


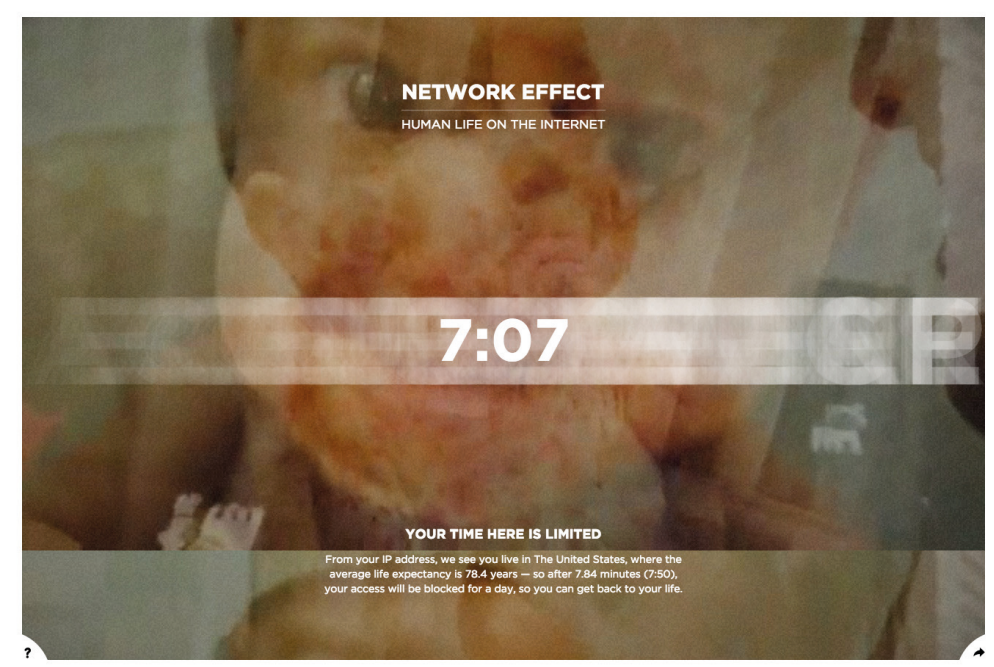

Abb. 7: Screenshot aus network effect (USA 2015 Pressebild; https://files.networkeffect.io/static/assets/vi/assets/press/images.zip?1.40).

Ein Countdown, der die verbleibende Verweildauer auf der Seite anzeigt, führt dazu, dass Inhalte nur in einem Modus des ständigen WeiterKlickens rezipiert werden. Eine Reflexion und Einordnung bzw. ein kritisches Durchdenken und Hinterfragen bleibt aus.

Selbstreflexiv und provokativ spielt die Intervention mit dem Phänomen, das der künstlerisch-dokumentarischen Intervention ihren Namen gab: dem Netzwerkeffekt.

Als 〈Netzwerkeffekt〉 wird in der neoklassischen Volkswirtschaftslehre die Tatsache bezeichnet, dass je mehr Menschen ein Gut oder eine Dienstleistung nutzen, der Wert dieses Gutes oder der Dienstleistung steigt. Das Internet ist paradigmatisch für den Netzwerkeffekt. Mit jeder Nutzerin bzw. jedem Nutzer, die bzw. der neue Informationen in das Netzwerk einspeist bzw. Daten hinterlässt, nimmt der Wert des Netzwerks als Ganzes zu. Inwieweit diese persönlichen Spuren jedoch wirklich einen Mehrwert in Form von Qualität, nicht nur Quantität der Daten erbringen, und inwieweit dies wirklich sinnstiftende Bedeutung schafft, Menschen über reine Konnektivität hinaus miteinander verbindet und eine sinnvolle Teilhabe an sozialen Netzwerken online und offline ermöglicht, schliesslich zur Entwicklung unseres Selbstbildes und unserer Sichtweise des Anderen beiträgt - all dies ist fraglich. 
Genau an diesem neuralgischen Punkt - der Sehnsucht und Suche nach unendlichen Wissenswelten, Partizipation, Subjektivierung und Kollektiverfahrung in und durch digitale Netzmedien in Anbetracht der ausbleibenden Befriedigung dieses Verlangens - setzt network effect als künstlerische Intervention an. So schreiben Harris und Hochmuth im Begleittext, dass sie mit network effect bewusst die Erfahrung destillieren und reinszenieren, die sich jeden Tag bei der Nutzung von Netzmedien einstellen: «The videos activate our voyeurism, the sound recordings tempt us with secrets, and the data promises a kind of omniscience, but all of it is a mirage - there is no one here to watch, there is no secret to find, and the data, which seems to be so important, is actually absurd [...] - full of tantalizing potential, but ultimately empty of life.» (Harris und Hochmuth 2015; http://networkeffect.io/epilogue)

Indem die Konfiguration also Gefühle provoziert, die sich häufig beim 〈Surfen〉 durch 〈das Netz〉 einstellen, macht sie erfahrbar, wie sinnentleert so manches Online-Verhalten ist. In network effect wird bewusst dass, was der amerikanische Pädagoge Dewey als «denkende Erfahrung» (1988) beschreibt, unterbunden wird, da das Design eine innere Distanzierung und kritische Reflektion verhindert. So wird es ver-un-möglicht, Erlebnisse zu verarbeiten, zu reflektieren und aus der Fülle von sinnlichen Eindrücken sinnstiftende Erfahrungen zu bilden. Ganzheitliche Denkerfahrungen erfordern Zeit und Fokussierung - sie sind, wie Niesyto formuliert, «das Zusammenspiel von sinnlichen Eindrücken, emotionaler Aufnahme und denkender Verarbeitung» (Niesyto 2012, 49). Für ihn - wie auch für Dewey - haben sie ästhetischen Charakter, sind aber zugleich «Wahrnehmungsprozesse, die in Verbindung mit Reflexionen zu einem gewissen Entwicklungsabschluss, zu einer Reifung kommen» (ebd.). Dabei geht es sowohl um «Prozesse der symbolischen Verarbeitung von Wahrnehmungen» als auch um «ästhetische Reflexivität - nicht um beliebiges, zusammenhangloses Antippen und Vorbeihuschen» (Niesyto 2012, 49). An dieser Stelle treffen Fragen der Ästhetik bzw. des Designs mit der Frage nach Bildungsprozessen im Digitalen zusammen - bzw. klafft die Diskrepanz zwischen dem 
Potenzial und den tatsächlich realisierten Verhaltens- und Handlungsweisen in digitalen Wissensräumen deutlich auf - wie in network effect auf frappierende Weise erlebbar und erfahrbar gemacht wird.

Aber nicht nur Subjektivierung auf individueller Ebene wird unterbunden. Wie bereits eingangs aufgezeigt, finden weder (Reifungsprozesse), wie sie Niesyto beschreibt, noch Deweys 〈denkende Erfahrung〉 in Isolation statt. Vielmehr sind all diese interdependenten und für umfassende kulturelle Bildungsprozesse essenziellen Erfahrungen, wie bereits diskutiert, relational: Es gehört dazu, «Widerstände zu überwinden, Spannungen auszuhalten, um Neues hervorzubringen» (Niesyto 2012, 49) - und dies geschieht nur in der Begegnung mit 〈dem Anderen〉. Mit dieser Reibung und dem 〈In-Kontakt-Treten〉 ist allerdings gerade nicht die oberflächliche Vernetzung 〈mit jedem an allem〉 gemeint, wie sie häufig in digitalen Netzwerken, insbesondere in social media networks, propagiert wird, denn es ist gerade nicht ein quantitatives 〈Mehr〉 an Partizipation, das zu einem (positiven) Netzwerkeffekt im Sinne eines inneren Wachstums und mehr Souveränität, Teilhabe und Teilnahme führt. Vielmehr tritt das ein, was in der Netzkritik als blosse Konnektivität bezeichnet wird (vgl. u. a. Apprich 2015; Lovink 1997) - ein Effekt, der wiederum Auswirkungen auf jegliche Art von Subjektivierungsprozessen hat. Und so führt network effect nicht zuletzt den Nutzenden die Kehrseite unreflektierter und totaler Vernetztheit, Verfügbarkeit, Enträumlichung und Entzeitlichung von Erleben vor Augen.

\section{Anstelle eines Fazits: Subjektivierung zwischen Individuation und Dividuation und die Überbrückung der Kluft zwischen dem Potenzial digitaler Wissensräume und Kulturen des Digitalen und ihrer aktuellen Realisierung}

Was lässt sich angesichts der Diagnose und nach Durchlaufen des zweiten Twists konstruktiv folgern? Beziehungsweise: Was könnten Wege sein, die - zumindest zunächst in Form von Erklärungsmodellen und theoretischen Konzepten - Perspektiven aufzeigen, wie man sich den Paradoxa und den Herausforderungen annähern kann, die Kulturen des Digitalen prägen? 
Ein möglicher Lösungsansatz bzw. zumindest eine theoretische Basis für weitere Überlegungen findet sich in einer Konzeption von Subjektivierung und Kollektivität im Sinne einer emanzipierten, souveränen Partizipation in und durch digitale Netzmedien, wie sie Ott beschreibt: Dividuation und Dividualität als eine Alternative zu Konzepten der Individualität und Kollektivität. Ott plädiert dabei für die Anerkennung der «cuts and divisions», die neben der umfassenden Vernetztheit die aktuelle conditio humana charakterisiert.

Dividuation betreffe dabei nicht nur den Menschen - vielmehr sei Dividuation ein Zustand, der sich durch alles Seiende ziehe - sowohl alles Technische als auch die Natur, sowohl die Künste als auch Wissensräume. Ott spricht von fluiden Wissensökologien, in denen epistemologische Differenzen fluid geworden seien. Diese Prozesse führten dazu, dass emotional wie digital kompetente, in digitalen Netzwerken interagierende Personen, die über die oben beschriebenen multimodalen literacies sowie sozial-emotionale Kompetenzen verfügen, in der Hinsicht umfassend digital souverän sein könnten, dass sie sich eben des Phänomens der Dividualität bewusst sind. Sie wissen um ihre «unconceived-of plasticity and mutability» ebenso wie um ihre "precarious psycho-physical stability» - oder, wie es Ott formuliert: Sie sind sich ihrer «qualified undividable multiple dividedness» bewusst (Ott 2018, 6) - und sie können diese kognitiv, emotional einkalkulieren und ihr Handeln dementsprechend ausrichten.

An diesem Punkt stellt sich zwangsläufig die Frage, wie ein Zugang zu diesen Überlegungen geschafften werden kann, die als eine Art MetaDiskurs essenziell sind, wenn es darum geht, ein selbstbestimmtes und zugleich offenes und experimentierfreudiges mediales Handeln in Kulturen des Digitalen zu ermöglichen.

Unter Umständen lässt sich ein Bewusstsein (und dies ist die Grundlage für alle weiteren Schritte) am ehesten durch ein Erleben der Effekte erlangen - hier des namengebenden network effects -, das heisst durch affektive, ästhetisch induzierte Erfahrungen, die über das kognitive Verständnis hinausweisen. Insofern führen paradoxe, irritierende und zum Teil auch frustrierende ästhetische Konfigurationen wie network effect als Anordnungen zur Selbsterfahrung trotz bzw. gerade aufgrund der ausbleibenden Befriedigung grundlegender menschlicher Bedürfnisse nach Bindung und 
Verbundenheit und obwohl eine eigene Position dazu gefunden wird, das eigene Verhalten zu hinterfragen. Zwar mag dieses Bewusstsein alleine natürlich nicht davor schützen, die eigene netzwerkfähige Hardware vor Trojanern und Schadsoftware zu schützen; es erübrigt nicht das ständige Aktualisieren der eigenen Sicherheitsstellungen; und es macht nicht davor gefeit, viral verbreiteten Falschmeldungen und fake news aufzusitzen; aber all die hier nur exemplarisch aufgezählten Massnahmen alleine hingegen machen wiederum auch nicht souveränes Handeln in einer tiefgreifreifend digital mediatisierten Gesellschaft aus und sind nicht der Kern dessen, was hier als kulturelle digitale Bildung beschrieben wurde, in der es vielmehr darum gehen sollte, ein Problembewusstsein für Herausforderungen zu schaffen.

Insofern finden sich in den Erfahrungen, die Konfigurationen wie network effect ermöglichen, die Anfänge von epistemischen 〈roten Fäden〉 in dem Gewirr von Implikationen, die zu Antworten auf zwei Fragen im Hinblick auf kulturelle Bildung in der digitalen Welt sein könnten, wie sie Jörissen (2017) stellt - zwei Fragen von hoher bildungstheoretischer Relevanz, fokussieren sie doch den Kern «ästhetisch-transformative[r] Aspekte von Bildungsprozessen unter Bedingungen der Digitalisierung»: Einerseits die Frage nach der «ästhetischen Reflexion des Digitalen, der digitalen Transformation von Kultur, der digitalen Praktiken in Bezug auf «Selbst und Welt> als Moment digitaler Bildung» - also die Frage, was es bedeutet, «in einer immer stärker von algorithmischen Logiken und datenbankkompatibler Weltproduktion abhängigen Kultur Subjekt zu sein?» (Jörissen 2017); und zum zweiten die Frage nach dem Potenzial digitaler Ästhetiken, nach dem impact von künstlerischen Manifestationen digitaler Kulturen «im Kontext postdigitaler Artikulationskulturen» - also die Frage nach der «Partizipation an der Reflexivität der Formdiskurse ästhetischer Praktiken» (Jörissen 2017).

Dass eine Reflexion des Digitalen in digitalen, mit digitalen und durch digitale Konfigurationen möglich ist - unter Umständen nur durch diese - sollte hier anhand der Überlegungen gezeigt werden; denn - und dies soll hier tentativ als Antwort auf Jörissens zweite Frage zur Diskussion 
gestellt werden: Vielleicht sind es gerade das Reflexive und die paradoxe künstlerische Intervention, die das sonst nur schwer Greifbare und Vermittelbare doch erfahrbar machen.

Um abschliessend zur Ausgangshypothese zurückzukehren: Was Konfigurationen, die über die Vermittlung primärer digital literacies hinausgehen, auszeichnet, ist ein zweiter twist: Indem sie Strategien wählen, die über die edukativ unbestreitbar effektiven Logiken prozeduraler Rhetorik, über immersive, personalisierte, interaktive Narration - kombiniert mit individuell konsultierbaren weiterführenden Materialien - hinausgehen, machen sie erfahrbar, was sich theoretisch nur schwer nachvollziehbar machen lässt.

\section{Literatur}

Aarseth, Espen J. 1997. Cybertext. Baltimore: Johns Hopkins University Press.

Apprich, Clemens. 2015. Vernetzt - Zur Entstehung der Netzwerkgesellschaft. Bd. 8. Digitale Gesellschaft. Bielefeld: transcript. https://doi. org/10.14361/9783839430453.

Aston, Judith, und Sandra Gaudenzi. 2012. «Interactive Documentary: Setting the Field». Studies in Documentary Film 6 (2), 125-39. https://doi.org/10.1386/ sdf.6.2.125_1.

Aston, Judith, und Stefano Odorico. 2018. «The Poetics and Politics of Polyphony: Towards a Research Method for Interactive Documentary». alphaville (15): 63-93. https://doi.org/10.33178/alpha.15.05.

Beck, Ulrich. 1992. Risikogesellschaft. Auf dem Weg in eine andere Moderne. Berlin: Suhrkamp.

Becker, Katrin. 2017. Choosing and Using Digital Games in the Classroom: A Practical Guide. Cham: Springer. https://doi.org/10.1007/978-3-319-12223-6.

Blossfeld, Hans-Peter und et al., Hrsg. 2018. Digitale Souveränität und Bildung: Gutachten. 1. Auflage. Münster: Waxmann. https://doi.org/10.25656/01:16569.

Bogost, Ian. 2007. Persuasive Games: The Expressive Power of Videogames. Cambridge: MIT Press.

Bogost, Ian. 2008. «The Rhetoric of Video Games». In The Ecology of Games: Connecting Youth, Games, and Learning, hrsg. von Katie Salen, 117-40. Cambridge, Mass: MIT Press. https://doi.org/10.1162/dmal.9780262693646.117.

Bolter, Jay David. 2009. The digital plenitude: The decline of elite culture and the rise of new media. Cambridge: MIT Press. https://doi.org/10.7551/mitpress/9440.001.0001. 
Couldry, Nick, und Andreas Hepp. 2013. «Conceptualizing Mediatization: Contexts, Traditions, Arguments». Communication Theory 23 (3): 191-202. https:// doi.org/10.1111/comt.12019.

Dewey, John. 1988/1934. Kunst als Erfahrung. Frankfurt am Main: Suhrkamp.

Fuchs, Mathias, Sonia Fizek, Paolo Ruffino, und Niklas Schrape, Hrsg. 2014. Rethinking Gamification. Lüneburg: Meson-Press. https://doi.org/10.14619/001.

Gaines, Jane, und Michael Renov, Hrsg. 1999. Collecting visible evidence. Minneapolis: University of Minnesota Press.

Gaylor, Brett. 2015. do not track. Produziert von arte, BR, National Film Board of Canada, Upian. http://donottrack-doc.com. http://networkeffect.io.

Gesellschaft für Informatik. 2020. «Schlüsselaspekte Digitaler Souveränität. Arbeitspapier». Unveröffentlichtes Manuskript, https://gi.de/meldung/gi-veroeffentlicht-arbeitspapier-zu-schluesselaspekten-digitaler-souveraenitaet.

Harris, Jonathan, und Greg Hochmuth. 2015. network effect. http://networkeffect. io.

Harris, Jonathan, und Greg Hochmuth. 2015. Works. network effect. http://number27.org/networkeffect.

Hepp, Andreas. 2018. «Von der Mediatisierung zur tiefgreifenden Mediatisierung». In Kommunikation - Medien - Konstruktion. Braucht die Mediatisierungsforschung den Kommunikativen Konstruktivismus?, herausgegeben von Jo Reichertz und Richard Bettmann, 27-45. Wissen, Kommunikation und Gesellschaft. Schriften zur Wissenssoziologie. Wiesbaden: Springer Fachmedien Wiesbaden. https://doi.org/10.1007/978-3-658-21204-9_2.

Hepp, Andreas. 2020. Deep mediatization. Key ideas in media and cultural studies. Abingdon, Oxon, New York, NY: Routledge. https://doi. org/10.4324/9781351064903.

Herzog, Werner, und Paul Cronin. 2002. Herzog on Herzog. London: Faber and Faber.

Holze, Jens, und Dan Verständig. 2020. «Medienbildung zwischen Subjektivität und Kollektivität - ein Problemaufriss». In Medienbildung zwischen Subjektivität und Kollektivität. Reflexionen im Kontext des digitalen Zeitalters, hrsg. von Jens Holze, Dan Verständig und Ralf Biermann, 1-14. Wiesbaden: Springer. https://doi.org/10.1007/978-3-658-31248-0.

Jenkins, Henry, Ravi Purushotma, Margaret Weigel, Katie Clinton, und Alice Robinson. 2009. Confronting the Challenges of Participatory Culture: Media Education for the 21st Century. Cambridge, MA: The MIT Press. https://doi. org/10.7551/mitpress/8435.001.0001.

Jenkins, Henry, Mizuko Itō, und danah boyd. 2016. Participatory Culture in a Networked Era: A Conversation on Youth, Learning, Commerce, and Politics. Cambridge, Malden, MA: Polity Press. https://doi.org/10.17192/ep2016.4.6302.

Jörissen, Benjamin. 2017. «Subjektivation und (ästhetische Freiheit) in der postdigitalen Kultur». https://doi.org/10.25529/92552.421. 
Kara, Selmin, und Camilla Møhring Reestorff. 2015. «Introduction: unruly documentary artivism». Studies in Documentary Film 9 (1): 1-9. https://doi.org/10.10 80/17503280.2014.1002665.

Kergel, David. 2018. Kulturen des Digitalen. Postmoderne Medienbildung, subversive Diversität und neoliberale Subjektivierung. Wiesbaden: Springer. https://doi. org/10.1007/978-3-658-20327-6.

Lovink, Geert. 2017. Im Bann der Plattformen: Die nächste Runde der Netzkritik. Digitale Gesellschaft. Bielefeld: transcript. https://doi.org/10.14361/9783839433683.

Manovich, Lev. 2001. The Language of New Media. Cambridge, Mass.: MIT Press.

Mihailidis, Paul. 2018. "Civic media literacies: re-Imagining engagement for civic intentionality». Learning, Media and TechnologY 43 (2): 152-64. https://doi.org/ 10.1080/17439884.2018.1428623.

Misterek, Fokko. 2017. «Digitale Souveränität: Technikutopien und Gestaltungsansprüche demokratischer Politik». Max-Planck-Institut für Gesellschaftsforschung, Köln. http://hdl.handle.net/10419/162561.

Müller, Jane, Mareike Thumel, Katrin Potzel, und Rudolf Kammerl. 2020. «Digital Sovereignty of Adolescents». MedienJournal 44 (1): 30-40. https://doi. org/10.24989/medienjournal.v44i1.1926.

Nash, Kate. 2022. Interactive Documentary. Theory and Debate. London, New York: Routledge. https://doi.org/10.4324/9781315208862.

Nichols, Bill. 1991. Representing Reality. Bloomington: Indiana University Press.

Nichols, Bill. 2008. «The Question of Evidence, the Power of Rhetoric and Documentary Film». In Rethinking documentary, hrsg. von Thomas Austin und Wilma deJong, 29-38. Maidenhead: Open University Press.

Nichols, Bill. 2016. Speaking Truths with Film: Evidence, Ethics, Politics in Documentary. Oakland, California: University of California Press.

Niesyto, Horst. 2012. "Bildungsprozesse unter den Bedingungen medialer Beschleunigung». In Raum, Zeit, Medienbildung. Untersuchungen zu medialen Veränderungen unseres Verhältnisses zu Raum und Zeit, hrsg. von Gerhard Chr. Bukow, Johannes Fromme und Benjamin Jörissen, 47-66. Wiesbaden: VS Verlag für Sozialwissenschaften. https://doi.org/10.1007/978-3-531-19065-5.

Ott, Michaela. 2018. Dividuations: Theories of Participation. Cham: Springer International Publishing. https://doi.org/10.1007/978-3-319-72014-2.

Pfadenhauer, Michaela, und Andreas Hepp. 2014. «Mediatisierte Partizipation? Kleine Formen der Beteiligung jenseits von Medienlogik». In Die Mediatisierung sozialer Welten: Synergien empirischer Forschung, hrsg. von Friedrich Krotz, Cathrin Despotović und Merle-Marie Kruse, 235-62. Wiesbaden: Springer. https://doi.org/10.1007/978-3-658-04077-2.

Salen, Katie, Hrsg. 2008. The Ecology of Games: Connecting Youth, Games, and Learning. The John D. and Catherine T. Macarthur Foundation Series on Digital Media and Learning. Cambridge, Mass: MIT Press. 
Spengler, Andreas. 2020. «Vernetzung als Subjektivierungsform: Konturierung einer Forschungsperspektive». In Medienbildung zwischen Subjektivität und Kollektivität: Reflexionen im Kontext des digitalen Zeitalters, hrsg. von Jens Holze, Dan Verständig und Ralf Biermann, 58-76. Wiesbaden: Springer VS. https://doi.org/10.1007/978-3-658-31248-0.

Thiele, Lena. 2015. netwars - Web Documentary. Produziert von Miiqo Studios UG und filmtank, in Zusammenarbeit mit ZDF/arte. http://www.netwars-project. com/de/webdoc.

Treanor, Mike, und Michael Mateas. 2009. «Newsgames - Procedural Rhetoric Meets Political Cartoons». In DiGRA \\&\\#3909 - Proceedings of the 2009 DiGRA International Conference: Breaking New Ground: Innovation in Games, PlaY, Practice and Theory, 1-8. Brunel University. http://www.digra.org/wp-content/uploads/digital-library/09300.09505.pdf.

Weber, Thomas. 2011. «Wissensvermittlung in medialer Transformation. Bemerkungen zu sich verändernden Wertmaßstäben in der bildungspolitischen Debatte». In Medien \& Bildung: Institutionelle Kontexte und kultureller Wandel, hrsg. von Torsten Meyer. 1. Aufl., 29-40. Medienbildung und Gesellschaft 20. Wiesbaden: Springer VS. https://doi.org/10.1007/978-3-531-92082-5.

Winston, Brian, Gail Vanstone, und Chi Wang. 2017. The act of documenting: documentary film in the 21st century. New York: Bloomsbury Academic. 